\title{
MicroRNA-33-5p inhibits cholesterol efflux in vascular endothelial cells by regulating citrate synthase and ATP-binding cassette transporter A1
}

Qiong Xie ${ }^{1}$, Jianqiang Peng ${ }^{1}$, Ying Guo ${ }^{1}$ and Feng $\mathrm{Li}^{2^{*}}$

\begin{abstract}
Background: A high level of total cholesterol is associated with several lipid metabolism disorders, including atherosclerosis and cardiovascular diseases. ATP-binding cassette (ABC) transporter A1 (ABCA1) and miR-33-5p play crucial roles in atherosclerosis by controlling cholesterol efflux. While citrate is a precursor metabolite for lipid and cholesterol synthesis, little is known about the association between citrate synthase (CS) and cholesterol efflux. This study investigated the role of the miR-33-5p/ABCA1/CS axis in regulating cholesterol efflux in vascular endothelial cells (VECs).
\end{abstract}

Materials and methods: VECS were treated with oxidized low-density lipoprotein cholesterol (ox-LDL), or pretreated with plasmids overexpressing CS, ABCA1, siRNAs against CS and ABCA1, and an miR-33-5p inhibitor. Cell apoptosis, cellular senescence-associated $\beta$-galactosidase activity, inflammation, and cholesterol efflux were detected.

Results: Treatment with ox-LDL decreased ABCA1 and CS levels and increased miR-33-5p expression and apoptosis in dose-dependent manners. In contrast, treatment with the miR-33-5p inhibitor and ABCA1 and CS overexpression plasmids inhibited the above-mentioned ox-LDL-induced changes. In addition, treatment with ox-LDL decreased cholesterol efflux, induced aging, and promoted the production of inflammatory cytokines (i.e., IL-6 and tumor necrosis factor TNF-a), as well as the expression of Bax and Caspase 3 proteins in VECs. All these changes were rescued by miR-33-5p inhibition and ABCA1 and CS overexpression. The inhibition of ABCA1 and CS by siRNAs eliminated the effects mediated by the miR-33-5p inhibitor, and knockdown of CS eliminated the effects of ABCA1 on VECs.

Conclusions: This study demonstrated the crucial roles played by the miR-33-5p/ABCA1/CS axis in regulating cholesterol efflux, inflammation, apoptosis, and aging in VECs, and also suggested the axis as a target for managing lipid metabolism disorders.

Keywords: ox-LDL, Citrate synthase, ABCA1, miR-33-5p, Vascular endothelial cells

*Correspondence: muzicn@csu.edu.cn

2 Departments of Cardiovascular Surgery, The Second Xiangya

Hospital of Central South University, middle Ren-Min Road No. 139,

Changsha 410011, Hunan, People's Republic of China

Full list of author information is available at the end of the article

\section{Introduction}

Lipid metabolism disorders are associated with several diseases, including hyperlipidemia, atherosclerosis, coronary heart disease, and cardiovascular diseases (CVDs). Lipid metabolism disorders are characterized by increased levels of total cholesterol (TC) and lowdensity lipoprotein (LDL) cholesterol and decreased levels of high-density lipoprotein (HDL) cholesterol [1, 2]. 
In addition to dysregulation of lipid metabolism, aberrant inflammatory responses are common in the diseases mentioned above, and are leading causes of mortality resulting from CVD and atherosclerosis [2-4]. Low density lipoproteins (LDLs) are lipoprotein particles that carry cholesterol into peripheral tissue cells, and can become oxidized to form oxidized LDLs. When LDL levels (especially ox-LDLs) become excessive, the cholesterol they carry accumulates on the arterial wall and ultimately causes arteriosclerosis. Oxidized low-density lipoproteins (ox-LDLs) participate in the formation and progression of lesions by eliciting lipid accumulation, and causing inflammation and toxic events $[5,6]$. It was reported that ox-LDLs combines with their major receptor (Lectin-like oxidized low-density lipoprotein receptor-1 [LOX-1]) to activate the NF-kB signaling pathway $[7,8]$. Ox-LDLs also affect the generation and utilization of endothelium-derived nitric oxide (NO), impair endothelium-dependent vascular diastolic function, and initiate the formation and development of AS plaques [7, 9-11].

Atherosclerosis is characterized by the formation of lipid plaques which mainly consist of lipid-laden foam cells transferred from macrophages, and therefore decrease the uptake of LDLs from blood vessel walls $[2,12]$. Several factors are associated with cholesterol efflux; these factors include the ATP-binding cassette (ABC) transporter A1 (ABCA1) protein family [13], miR-33-5p [14-16], inflammatory factors such as interleukin (IL)- 6 and tumor necrosis factor (TNF)- $\alpha$ [17], and the 3-hydroxy-3-methylglutaryl coenzyme A reductase (HMGCR) enzyme. ABCA1 plays a crucial role in removing excess lipids by initiating reverse cholesterol transport (RCT) and mediating the transport of intracellular free cholesterol and phospholipids from peripheral tissues to lipid-poor apolipoprotein A I (ApoA1) [18]. In addition, ABCA1 also promotes the production of athero-protective HDL cholesterol [18, 19]. MiR-33-5p targets ABCA1, and knockdown of miR-33-5p decreases the levels of total cholesterol (TC) and TNF- $\alpha$, and promotes cholesterol efflux from foam cells $[13,20]$. It has been reported that the inflammatory cytokines IL- 6 and TNF- $\alpha$ can suppress ABCA1 expression and thereby inhibit cholesterol efflux [17].

Citrate is a precursor metabolite for lipid and cholesterol synthesis, and accumulation of exogenous citrate promotes inflammation in visceral adipose tissue [21]. Lower rates of citrate synthase (CS) activity have been reported to be associated with increased levels of cleaved caspase-3 and lipotoxicity in C1C12 muscle cells [22]. Our previous experiments showed that CS is a direct target of miR-33-5p. However, there have been no reports of CS activity being associated with cholesterol efflux or lipid metabolism disorders such as atherosclerosis or CVD.

This current study investigated how the miR-33-5p/ ABCA1/CS axis helps to control cholesterol efflux in vascular endothelial cells (VECs). VECs were treated with ox-LDLs and pretreated with a miR-33-5p inhibitor, ABCA1 and CS expression plasmids, or with siRNAs. The effects on cell apoptosis, cellular cholesterol efflux, and inflammatory cytokines (IL-6 and TNF- $\alpha$ ) that occurred in response to gene modulation were detected. Our results provide novel insights into potential mechanisms associated with lipid metabolism.

\section{Materials and methods}

\section{Cells, culture conditions, and treatments}

Human VECs (CRL-1730, ATCC, Manassas, VA, USA) were maintained in DMEM (Hyclone, Logan, UT, USA) containing 1\% antibiotics (penicillin-streptomycin), 10\% FBS (Sigma-Aldrich; Merck KGaA, Darmstadt, Germany), with or without oxidized low-density lipoproteins (ox-LDLs) at a low $(50 \mu \mathrm{g} / \mathrm{mL})$, moderate $(100 \mu \mathrm{g} /$ $\mathrm{mL})$ or high concentration $(200 \mu \mathrm{g} / \mathrm{mL})$ [23]. The oxLDLs (Shanghai Jingke Chemical Technology Co., LTD, Shanghai, China) were dissolved in PBS, and then diluted to different concentrations. The VECs were incubated in DMEM at $37{ }^{\circ} \mathrm{C}$ in a $5 \% \mathrm{CO}_{2}$ atmosphere, and then induced with ox-LDLs for $48 \mathrm{~h}$.

\section{Plasmids and cell transfections}

Plasmids overexpressing ABCA1 (OE-ABCA1) and CS (OE-CS) were constructed using the pcDNA3.1 vector (Genechem Co. Ltd., Shanghai, China) and full CDS sequence cloning. The polymerase chain reaction (PCR) was used to clone the ABCA1 or CS coding sequences into the plasmid pcDNA3.1 vector. The pcDNA3.1 vector without the $A B C A 1$ and $C S$ genes served as a negative control. pcDNA3.1-ABCA1 or pcDNA3.1-CS was transfected into VECs (6-well plates, $\left.1.0 \times 10^{6} / \mathrm{cm}^{2}\right)$. Short interfering RNAs (siRNAs) against human $A B C A 1$ and $C S$ genes, miR-33-5p mimics (B01001), inhibitors (B03001) (chemically modified inhibitors that specifically targeted miR-33-5p), and scramble sequences were obtained from GenePharma Corporation (Shanghai, China). Cell transfections were performed using Lipofectamine 2000 (Invitrogen, Carlsbad, CA, USA) according to the manufacturer's instructions. Cells transfected with the empty pcDNA3.1 vector or scrambled sequences served as negative controls (NCs) for overexpression and for siRNA, mimics or inhibitors, respectively. Cells were transfected with the different agents for $48 \mathrm{~h}$ before harvesting. Each experiment was repeated 3 times. 


\section{Dual-luciferase reporter assay}

Possible interactions between miR-33-5p and ABCA1 or CS were predicated using TargetScanHuman (http:// www.targetscan.org/vert_72/), and subsequently verified by using the dual-luciferase reporter system. Luciferase vectors containing miR-33-5p binding sites (wild type, WT; and mutant, MUT, 7 base substitutions) in the $3^{\prime}$ UTR regions of $A B C A 1$ or $C S$ genes were constructed using XhoI and NotI restriction enzymes; Thermo Fisher Scientific Inc., Waltham, MA, USA) and a psiCHECK-2 expression vector (Promega, Madison, WI, USA). Cell transfections were performed using Lipofectamine 2000 (Invitrogen).

\section{Enzyme-linked immuno sorbent assay (ELISA)}

Cell culture samples were collected and prepared for ELISA. The levels of CS, interleukin-6 (IL-6), and tumor necrosis factor- $\alpha$ (TNF- $\alpha)$ in the samples were detected using ELISA kits (Elabscience Biotechnology Co. Ltd., Wuhan, China). A microplate reader (Thermo Fisher Scientific Inc.) was used to measure optical density (OD) at $450 \mathrm{~nm}$.

\section{Cholesterol efflux determination}

The total cholesterol efflux in culture medium and cell lysate was measured using a Cholesterol Efflux Fluorometric Assay Kit (BioVision, San Francisco, CA USA) according to the manufacturer's protocol [24]. The fluorescence-labeled cholesterol was washed with $200 \mu \mathrm{L}$ of phenol red-free, serum-free RPMI medium and then incubated in serum-free RPMI medium containing cholesterol acceptor ApoA 1 (50 $\mu \mathrm{g} /$ well $)$ for $16 \mathrm{~h}$ at $37^{\circ} \mathrm{C}$. The total fluorescence intensity at $48 \mathrm{~h}$ post-transfection or treatment was detected at excitation and emission wavelengths of $482 \mathrm{~nm}$ and $515 \mathrm{~nm}$, respectively. Cholesterol efflux was calculated as: $100 \% \mathrm{x}$ media fluorescence intensity/total fluorescence intensity.

\section{Flow cytometric analysis and aging assay}

Cell apoptosis was detected by flow cytometry. Cells treated or transfected with different agents for $48 \mathrm{~h}$ were harvested and fixed. Cell apoptosis was detected by using an Annexin V-FITC/PI Double Staining Kit (BD Biosciences, San Jose, CA, USA) according to the manufacturer's instructions. The percentages of apoptotic VECs were analyzed using a BD FACS Calibur flow cytometer (BD Biosciences). Aged cells were detected by using a $\beta$-Galactosidase staining kit (Jiancheng Biological Company, Nanjing, China) according to the manufacturer's recommended protocol. Senescence-associated $\beta$-galactosidase (SA- $\beta$-Gal) in aged cells was detected as previously described [25]. Cellular images were captured using a microscope (Motic, Xiamen, China). Each experiment was repeated 3 times.

\section{Quantitative real-time PCR (qRT-PCR) analysis}

Total cellular RNA was extracted using TRIzol reagent (Invitrogen), and first strand cDNA was synthesized using a Bestar qPCR RT Kit (DBI Bioscience, Shanghai, China). MiR-33-5p expression was achieved by reverse transcription that was performed using a stem-loop primer. A DBI Bestar ${ }^{\circledR}$ SYBR Green qPCR master mix kit (DBI Bioscience) was used for qRT-PCR amplification conducted on an Mx3000P Agilent Stratagene PCR machine (Agilent, Santa Clara, CA, USA). The primers were synthesized by Sangon Biotechnology Co., Ltd. (Shanghai, China; Table 1). The following conditions were used for amplification: $95^{\circ} \mathrm{C}$ for $2 \mathrm{~min}$; followed by 40 cycles of $94{ }^{\circ} \mathrm{C}$ for $20 \mathrm{~s}, 58^{\circ} \mathrm{C}$ for $20 \mathrm{~s}$, and $72{ }^{\circ} \mathrm{C}$ for $20 \mathrm{~s}$. The relative levels of mRNA or miR-33-5p expression were calculated using standard $2^{-\triangle \triangle \mathrm{Ct}}$ methods. U6 and GAPDH served as internal reference genes.

\section{Western blot analysis}

The total cellular proteins were extracted using RIPA lysis buffer (Beyotime, Shanghai, China), and the protein concentration in each extract was determined using a protein quantification kit (Thermo Fisher Scientific). An aliquot of total protein from each extract was separated by $10 \%$ SDS-PAGE (Sangon Biotechnology Co., Ltd), and the protein bands were transferred onto PVDF membranes (Millipore Corporation, Burlington, MA, USA), which were subsequently blocked with $5 \%$ skim milk powder (Beyotime). Next, the membranes were incubated with specific primary antibodies against HMGCR (1: 1000;

Table 1 Primers used for quantitative real time PCR analysis

\begin{tabular}{ll}
\hline Gene & Primer sequence $\mathbf{5}^{\prime}$-3' $^{\prime}$ \\
\hline Hsa-miR-33 & F: ACACTCCAGCTGGGGTGCATT \\
& GTAGTTGCAT \\
& RT: CTCAACTGGTGTCGTGAGTC \\
& GGCAATTCAGTTGAGTGAATG \\
All & R: CTCAACTGGTGTCGGGA \\
ABCA1 & F: ACCCACCCTATGAACAACATGA \\
CS & R: GAGTCGGGAACGGAACAGG \\
HMGCR & RACTGCTACCCAAGGCTAAGG \\
U6 CTTTGAGAGCCAAGATACCTGT & F: AACTGCTACCCAAGGCTAAGG \\
R: CTAAAATTGCATTCCACGAGC \\
FAPDH
\end{tabular}

$F$ forward primer, $R$ revise primer, $R T$ reverse transcription 
ab174830, Abcam, Cambridge, UK), ABCA1 (1: 1000; \#96292, CST, Danvers, MA, USA), CS (1: 1000; \#14309, CST), Caspase 3 (1: 1000; \#14220, CST), Bax (1: 1000; \#5023, CST), Bcl-2 (1: 1000; \#4223, CST), and GAPDH (1: 2500; ab9485, Abcam), and subsequently incubated with a secondary antibody (HRP Goat anti-rabbit/ mouse IgG, 1: 20,000; Boster, Wuhan, China). The immunostained protein bands were detected using enhanced chemiluminescence luminous fluid (Millipore), and band staining intensity was analyzed using a scanner (Microtek, Carson, CA, USA).

\section{Statistical analysis}

The GraphPad Prism 8.0 (GraphPad, La Jolla, USA) was used for data analysis. Experiments were repeated in three times, all data are expressed as a mean value \pm standard deviation (SD). The distribution normality of all data was verified prior before analysis by one-way ANOVA. Continuous data obtained from multiple groups was analyzed by one-way ANOVA followed by Tukey's post hoc test. A P-value $<0.05$ was considered to be statistically significant, $\mathrm{P}$-value $<0.01$ was considered as obvious statistical significance.

\section{Results}

Ox-LDL increased miR-33-5p levels and decreased ABCA1 and CS levels

Administration of ox-LDL to VECs induced a dosedependent inhibition of both ABCA1 and CS expression and increased miR-33-5p expression (Fig. 1A-C). HMGCR expression was only increased by the high concentration of ox-LDL. Ox-LDL also induced dosedependent apoptosis in VECs (Fig. 1D, E). These data suggest that ox-LDL has a dose-dependent effect on both ABCA1 and CS expression, as well as on VEC apoptosis, whereas no such dose-dependent effects were found for HMGCR. Accordingly, we selected the moderate dose of ox-LDL for use in further experiments.

\section{Suppression of miR-33-5p impeded ox-LDL-induced changes in VECs}

Inhibition of miR-33-5p impeded the ox-LDL-induced expression of miR-33-5p and HMGCR, and significantly increased the levels of both ABCA1 and CS expression $(p<0.01$, Fig. 2A, B). The decreases in CS content and cholesterol efflux in VECs induced by ox-LDL were recovered by treatment with the miR-33-5p inhibitor $(p<0.01$, Fig. 2C, D). The ox-LDL-induced increases in

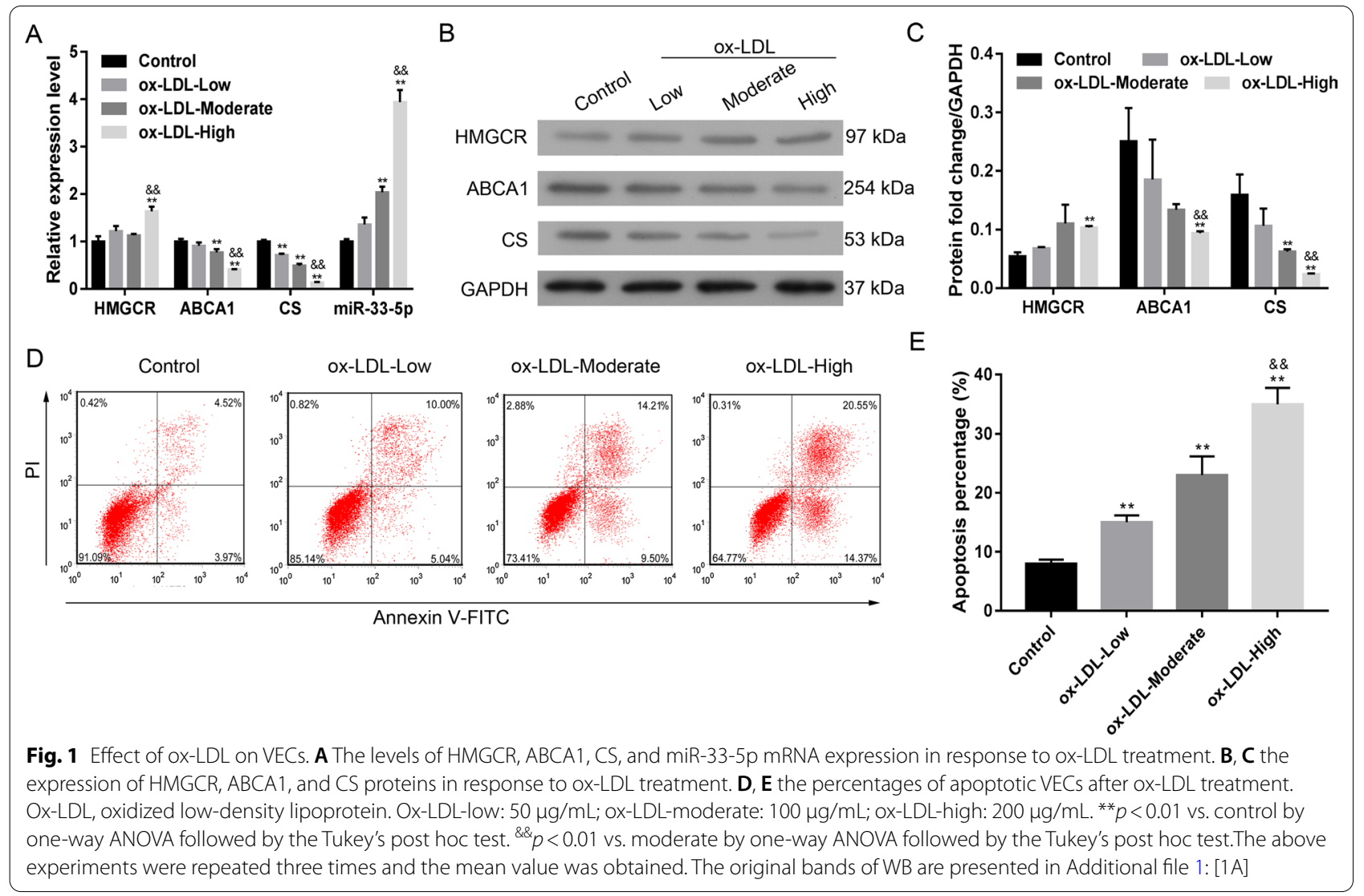



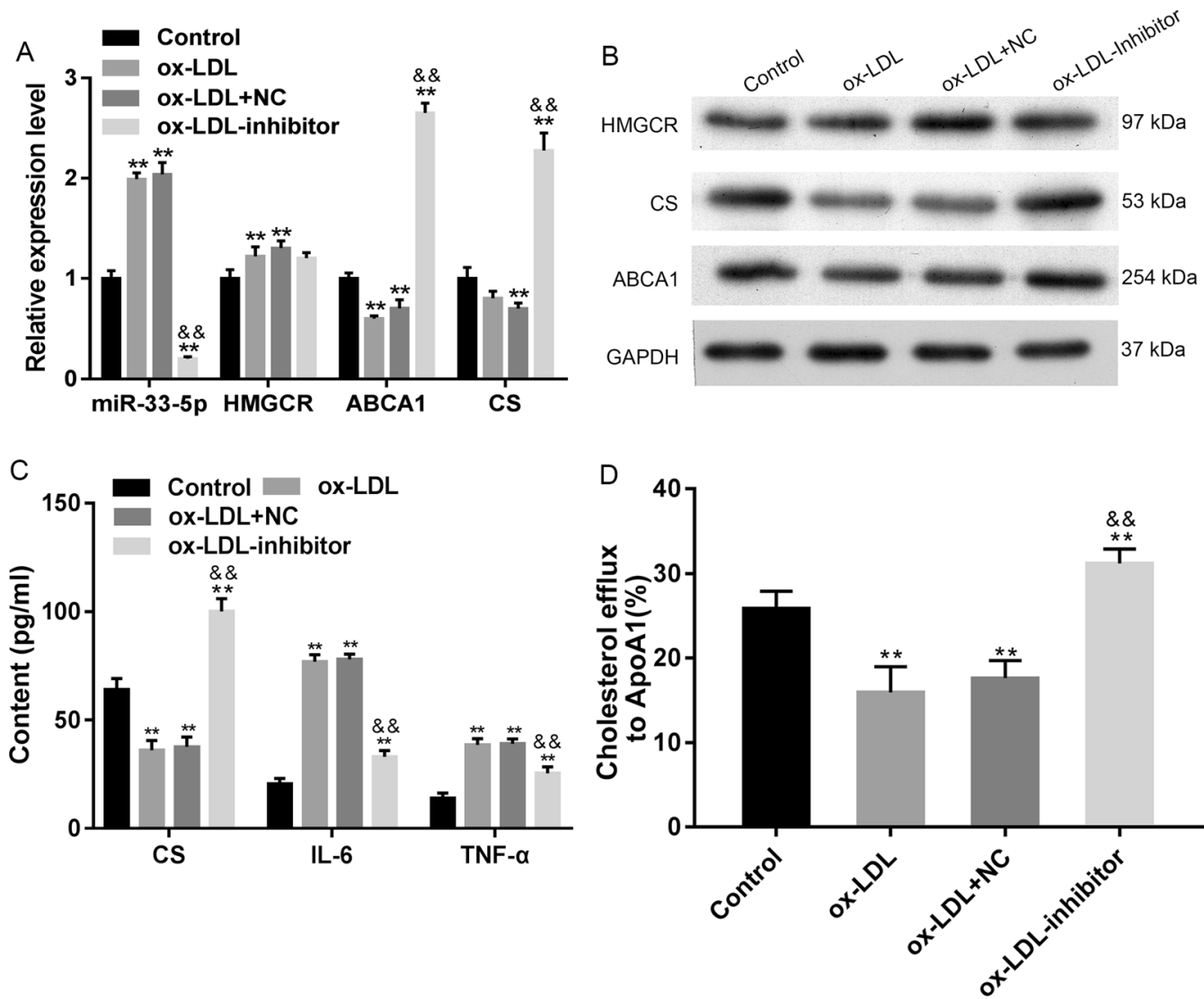

Fig. 2 Effect of miR-33-5p inhibition on VECs. A the relative expression profiles of miR-33-5p, HMGCR, ABCA1, and CS mRNA. B The expression of HMGCR, ABCA1, and CS proteins in response to miR-33-5p inhibition. The original bands of WB are presented in Additional file 1: [1B]. C the levels of CS, IL-6, and TNF-a in VECs. D the cellular cholesterol efflux to ApoA 1 (50 $\mu \mathrm{g} /$ well) in VECs. Ox-LDL, oxidized low-density lipoprotein.NC, inhibitor negative control. ${ }^{* *} p<0.01$ vs. control by one-way ANOVA followed by Tukey's post hoc test. ${ }^{\& \&} p<0.01$ vs. ox-LDL by one-way ANOVA followed by Tukey's post hoc test. The above experiments were repeated three times and the mean value was obtained

cellular IL-6 and TNF- $\alpha$ levels were attenuated by the miR-33-5p inhibitor $(p<0.01$, Fig. $2 \mathrm{C})$, as well as the levels of cell apoptosis and SA- $\beta$-gal activity (Fig. 3A-D). Bax and Caspase 3 expression were decreased by the miR-33-5p inhibitor, while Bcl-2 expression was upregulated by the miR-33-5p inhibitor (Fig. 3D). These data showed that miR-33-5p inhibition impeded ox-LDLinduced inflammation, apoptosis, and aging of VECs by increasing $\mathrm{ABCA} 1$ and $\mathrm{CS}$ expression and cholesterol efflux.

\section{$A B C A 1$ and $C S$ overexpression impeded ox-LDL-induced changes in VECs}

We next examined whether overexpression of ABCA1 and CS in VECs would impede the ox-LDL-induced inhibition of $\mathrm{ABCA} 1$ and CS (Fig. 4A, B, E). Our data showed that overexpression of ABCA1 significantly increased both CS and ABCA1 levels, while overexpression of CS only significantly increased CS levels $(p<0.01$, Fig. 4A, B). The decreases in CS content and cholesterol efflux, as well as the increases in IL-6 and TNF- $\alpha$ levels in VECs induced by ox-LDL were rescued by ABCA1 and CS overexpression $(p<0.01$, Fig. $4 \mathrm{~B}, \mathrm{C})$. Both ABCA1 and CS expression, and especially the former, decreased ox-LDL-induced cell apoptosis and SA- $\beta$-gal activity (Fig. 5A, B). Cellular SA- $\beta$-gal activity, apoptosis, and Bax and Caspase 3 expression were all increased by ox-LDL administration and rescued by ABCA1 and CS expression (Figs. 4, 5), while Bcl-2 expression was decreased by ox-LDL administration and rescued by ABCA1 and CS expression (Fig. 5D).

\section{MiR-33-5p targeted ABCA1 and CS}

The predicted target interactions of miR-33-5p with ABCA1 and CS were verified by dual-luciferase reporter assays (Fig. 6A, B). Further siRNA experiments showed that siCS and siABCA1 inhibited the miR-33-5p inhibitor-induced expression of $\mathrm{ABCA} 1$ and $\mathrm{CS}$ (Fig. 7A, B). Cell populations that were co-transfected with the miR-33-5p inhibitor plus siCS or siABCA1 had higher 

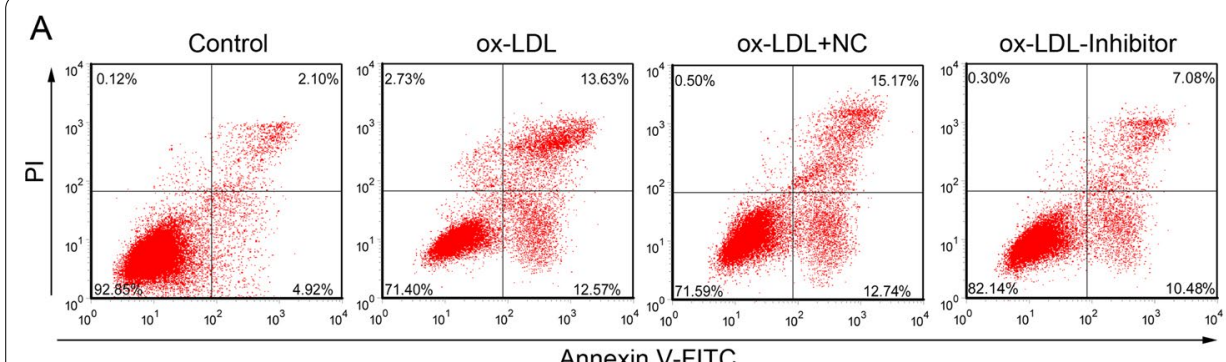

B

C

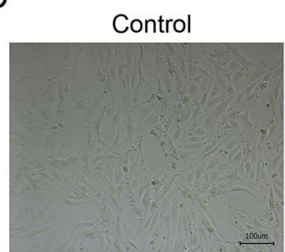

ox-LDL
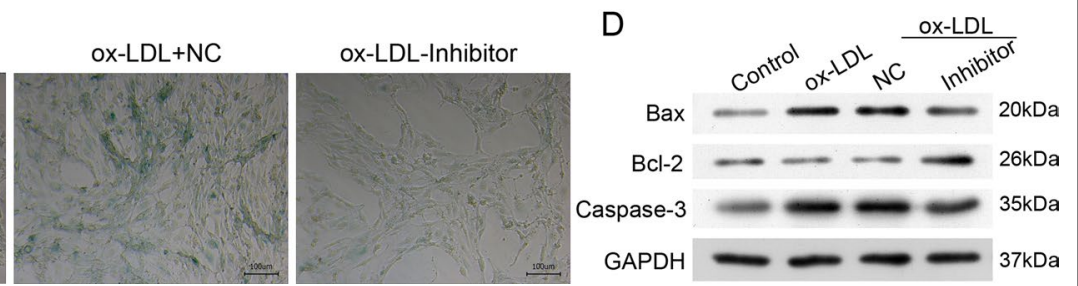

Fig. 3 Influence of miR-33-5p inhibition on apoptosis and aging of VECS. A, B the percentages of apoptotic VECs. C The inhibitory effect of miR-33-5p on senescence-associated $\beta$-galactosidase (SA- $\beta$-gal) activity in VECs. The repeated results were shown in Aditional file 2: Supplementary Figure 3. D The expression of apoptosis-related proteins. Ox-LDL, oxidized low-density lipoprotein. NC, inhibitor negative control. The original bands of WB are presented in Additional file 1: [1C]. ${ }^{* *} p<0.01$ vs. control by one-way ANOVA followed by Tukey's post hoc test. ${ }^{\& \&} p<0.01 \mathrm{vs}$. ox-LDL by one-way ANOVA followed by Tukey's post hoc test. The above experiments were repeated three times and the mean value was obtained
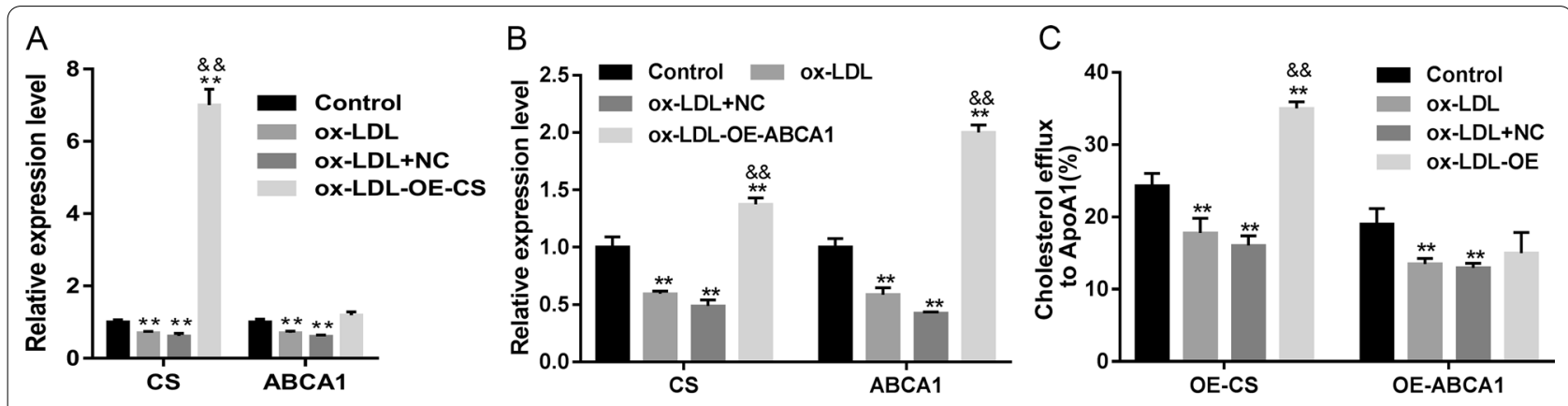

$\mathrm{D}$

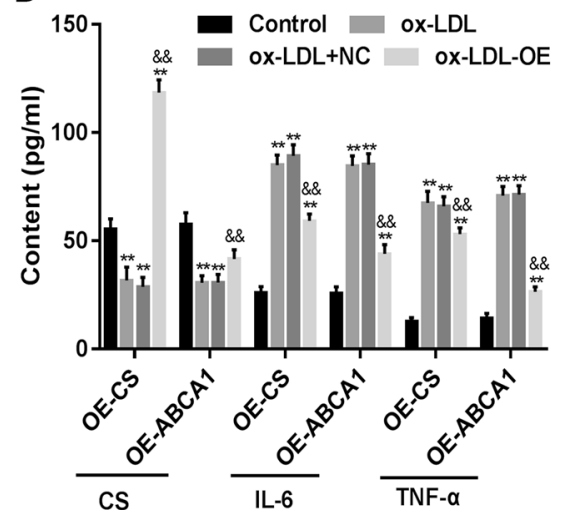

E

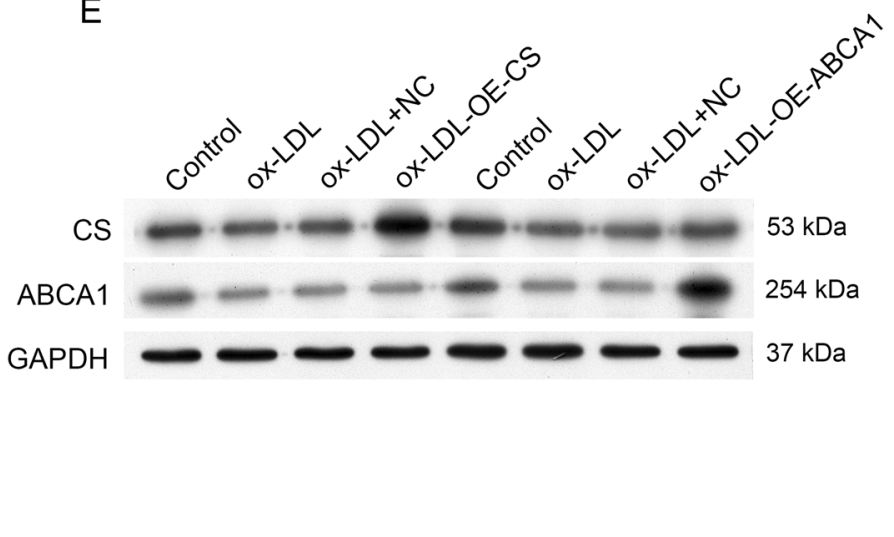

Fig. 4 Effect of CS and ABCA1 overexpression on cellular secretion. A, B the overexpression of CS and ABCA1 impeded ox-LDL-reduced expression. C The cellular cholesterol efflux to ApoA $1(50 \mu \mathrm{g} /$ well) in VECs. D The levels of CS, IL-6, and TNF-a in VECs. E The levels of CS and ABCA1 protein expression in VECs. Ox-LDL, oxidized low-density lipoprotein. NC, overexpression negative control. The original bands of WB are presented in Additional file 1: [1D]. ${ }^{* *} p<0.01$ vs. control by one-way ANOVA followed by Tukey's post hoc test. ${ }^{\& \&} p<0.01 \mathrm{vs}$. ox-LDL by one-way ANOVA followed by Tukey's post hoc test. The above experiments were repeated three times and the mean value was obtained 


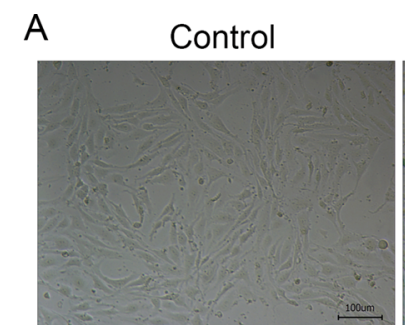

Control

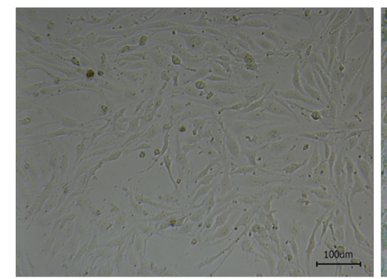

B

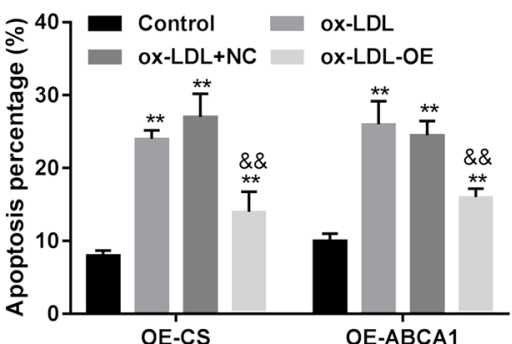

ox-LDL

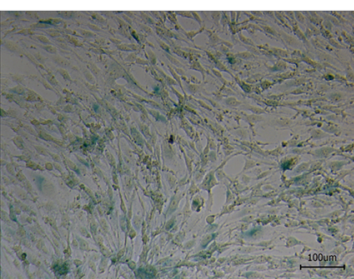

ox-LDL

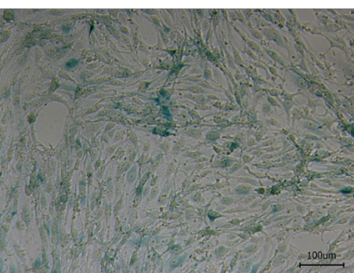

ox-LDL+NC

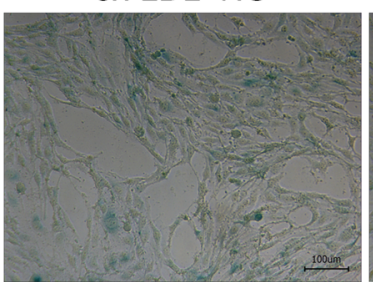

$\mathrm{ox}-\mathrm{LDL}+\mathrm{NC}$

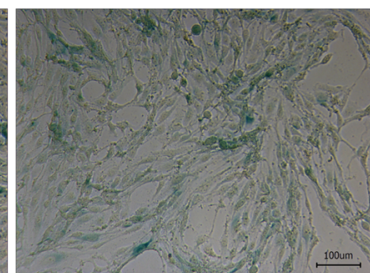

OX-LDL-OE-CS

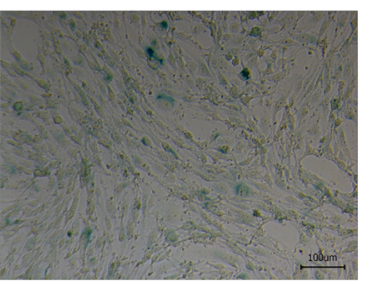

ox-LDL-OE-ABCA1

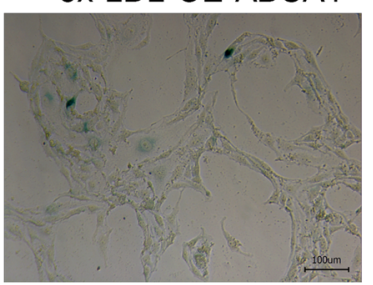

C

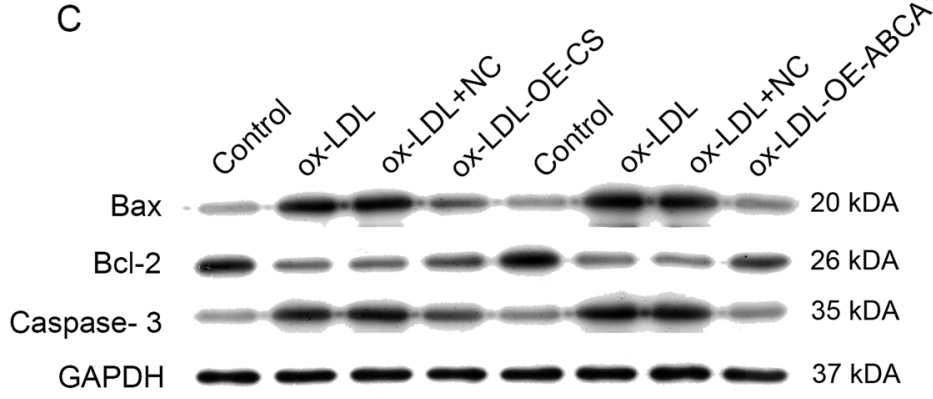

Fig. 5 Effect of CS and ABCA1 overexpression on cellular apoptosis and aging. A the levels of senescence-associated $\beta$-galactosidase (SA- $\beta$-gal) activity in VECs. The repeated results were shown in Aditional file 2: Supplementary Figure 4. B the percentages of apoptotic VECs. $\mathbf{C}$ the expression of apoptosis-related proteins. Ox-LDL, oxidized low-density lipoprotein. NC, overexpression negative control. The original bands of WB are presented in Additional file 1: [1E]. ${ }^{* *} p<0.01$ vs. control by one-way ANOVA followed by Tukey's post hoc test. ${ }^{\& \&} p<0.01$ vs. ox-LDL by one-way ANOVA followed by Tukey's post hoc test. The above experiments were repeated three times and the mean value was obtained

A

miR-33-5p-CS

1356-1362 of CS 3' UTR 5' ...GGUUGUUCUGUAUAAAAUGCAAA.....3' hsa-miR-33a/b-5p $\quad 3^{\prime} \quad$ CGUUACGUUGUCGUUACGUG

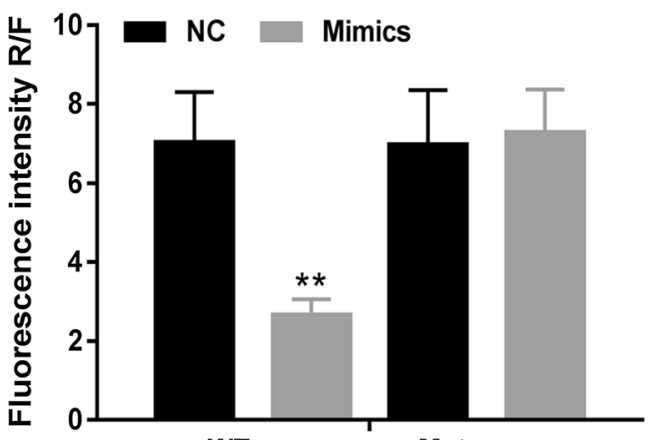

WT
B

miR-33-5p-ABCA1

135-142 of ABCA1 3'UTR 5' ...UguacugauACUAUUCAAUgCAA.. hsa-miR-33a/b-5p 3' ACGUUACGUUGAUGUUACGUG

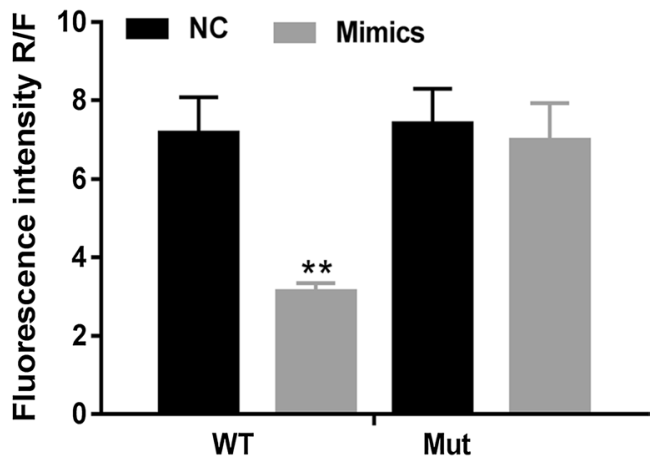

Fig. 6 Dual-luciferase reporter assay results. A the prediction and determination of interaction between miR-33-5p and CS. B The predication and determination of interaction between miR-33-5p and ABCA1. NC, mimic negative control. WT, wild type. Mut, mutation. ${ }^{* *} p<0.01$ vs. control by one-way ANOVA followed by Tukey's post hoc test. The above experiments were repeated three times and the mean value was obtained 

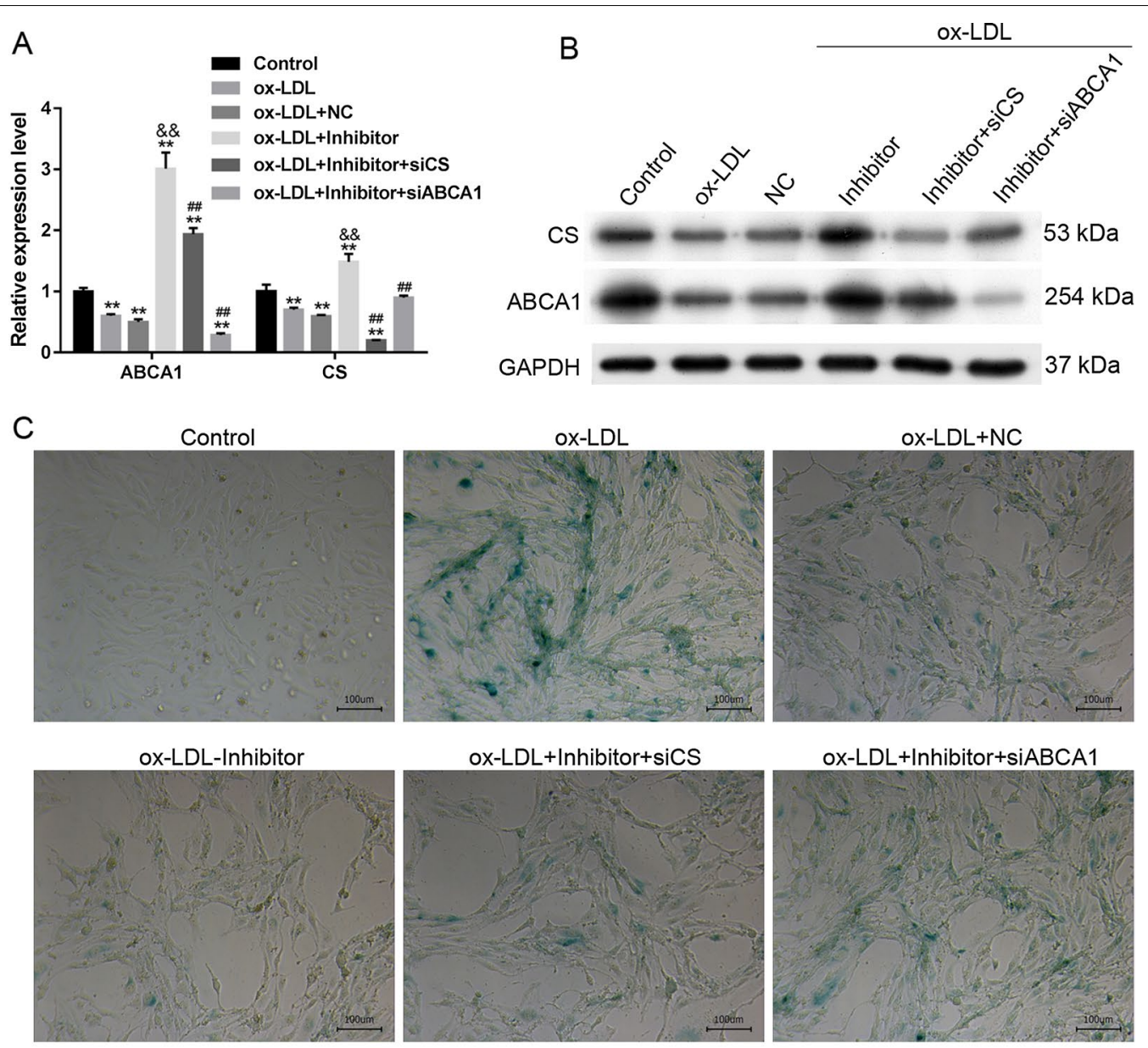

ox-LDL+Inhibitor+siABCA1

D

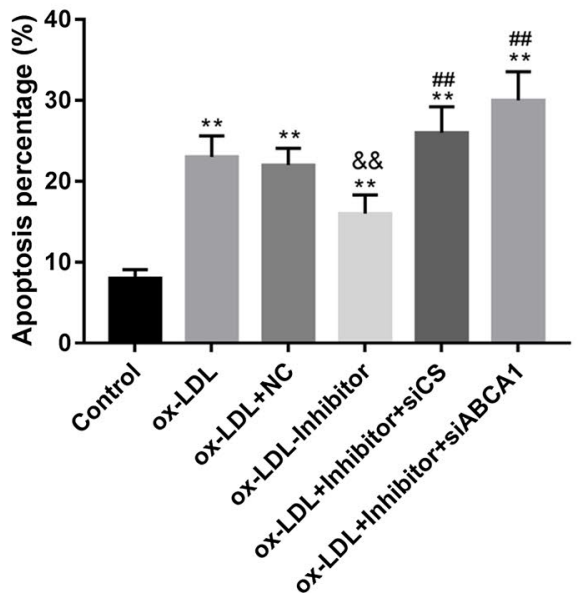

E

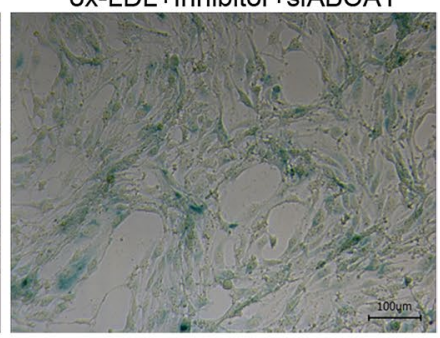

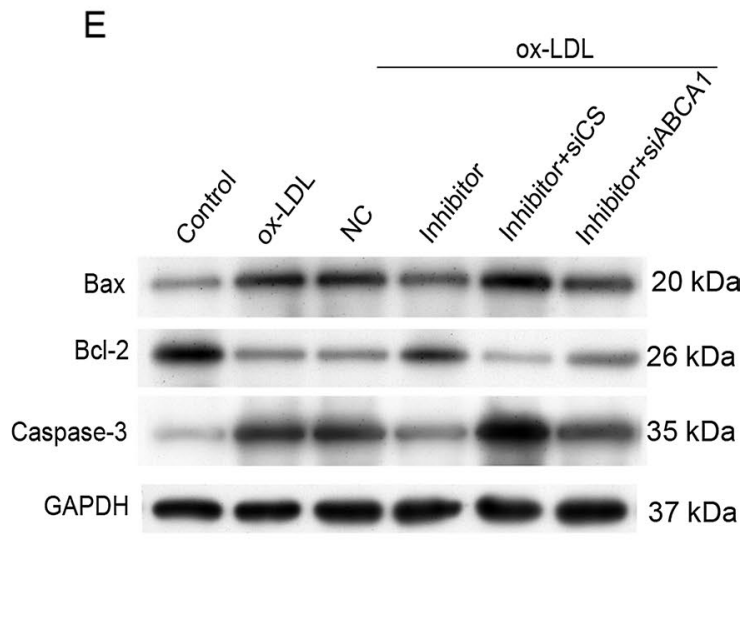

Fig. 7 Antagonistic effect of $C S$ and $A B C A 1$ overexpression on miR-33-5p inhibition. A, B The levels of ABCA1 and CS mRNA and protein expression in response to transfections. C The levels of senescence-associated $\beta$-galactosidase (SA- $\beta$-gal) activity in VECs. D The percentages of apoptotic VECs. The repeated results were shown in Aditional file 2: Supplementary Figure 5. E The expression of apoptosis-related proteins. Ox-LDL, oxidized low-density lipoprotein. NC, overexpression negative control. The original bands of WB are presented in Additional file 1: [2A and $2 B] .{ }^{* *} p<0.01 \mathrm{vs}$. control by one-way ANOVA followed by Tukey's post hoc test. ${ }^{\& \&} p<0.01 \mathrm{vs}$. ox-LDL by one-way ANOVA followed by Tukey's post hoc test. ${ }^{\# \#} p<0.01$ vs. OE-ABCA1 by the one-way ANOVA followed by Tukey's post hoc test. The above experiments were repeated three times and the mean value was obtained 
levels of SA- $\beta$-gal activity (Fig. 7C), higher percentages of apoptotic cells (Fig. 7D) and higher levels of Bax and Caspase 3 expression (Fig. 7E) when compared to cells transfected with the miR-33-5p inhibitor alone $(p<0.01)$. Bcl-2 expression was decreased by treatment with oxLDLs and rescued by the miR-33-5p inhibitor, which was further inhibited by siCS and siABCA1 transfection (Fig. 7E). These experiments demonstrated the interaction of miR-33-5p with ABCA1 and CS.

\section{Knockdown of CS antagonized ABCA1-induced changes in VECs}

We confirmed that ABCA1 overexpression induced CS expression (Fig. 8A, B) and cholesterol efflux (Fig. 8C), and that ABCA1 inhibited inflammation (Fig. 8D), apoptosis (Fig. 9A), and SA- $\beta$-gal activity (Fig. 9C); furthermore, these changes could be rescued by siCS transfection. The decreases in Bax and Caspase 3 expression caused by $\mathrm{ABCA} 1$ overexpression were recovered by
siCS; however, siCS decreased Bcl-2 expression (Fig. 9B). These results indicated that the ABCA1/CS axis was essential for inflammation, cholesterol efflux, apoptosis, and aging in VECs.

\section{Discussion}

Low density lipoproteins (LDLs) are lipoprotein particles capable of carrying cholesterol into peripheral tissue cells, where it can be oxidized to form oxidized LDL. When the levels of LDLs, and especially ox-LDLs, become excessive, the cholesterol they carry will accumulate on the arterial wall and eventually cause arteriosclerosis. High density lipoproteins (HDLs) have received more attention as a preventive factor of atherosclerosis because they can export cholesterol and promote cholesterol metabolism. Furthermore, HDLs can help to atherosclerosis and protect against coronary heart disease. Many factors are known to be associated with cholesterol efflux, such as ABCA1 and miR-33-5p [14, 15]. Our
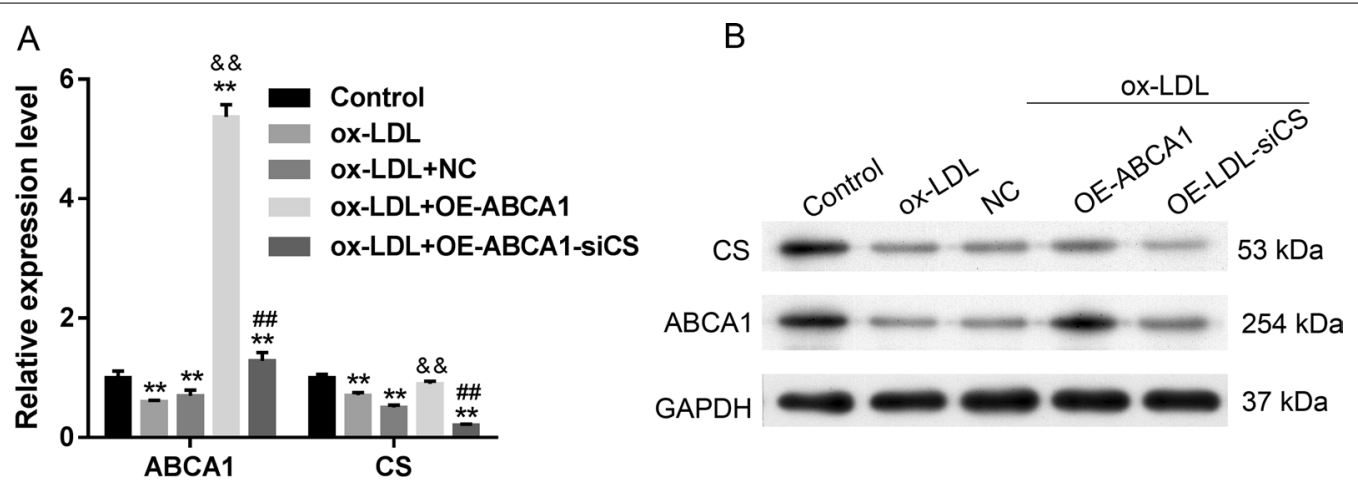

C
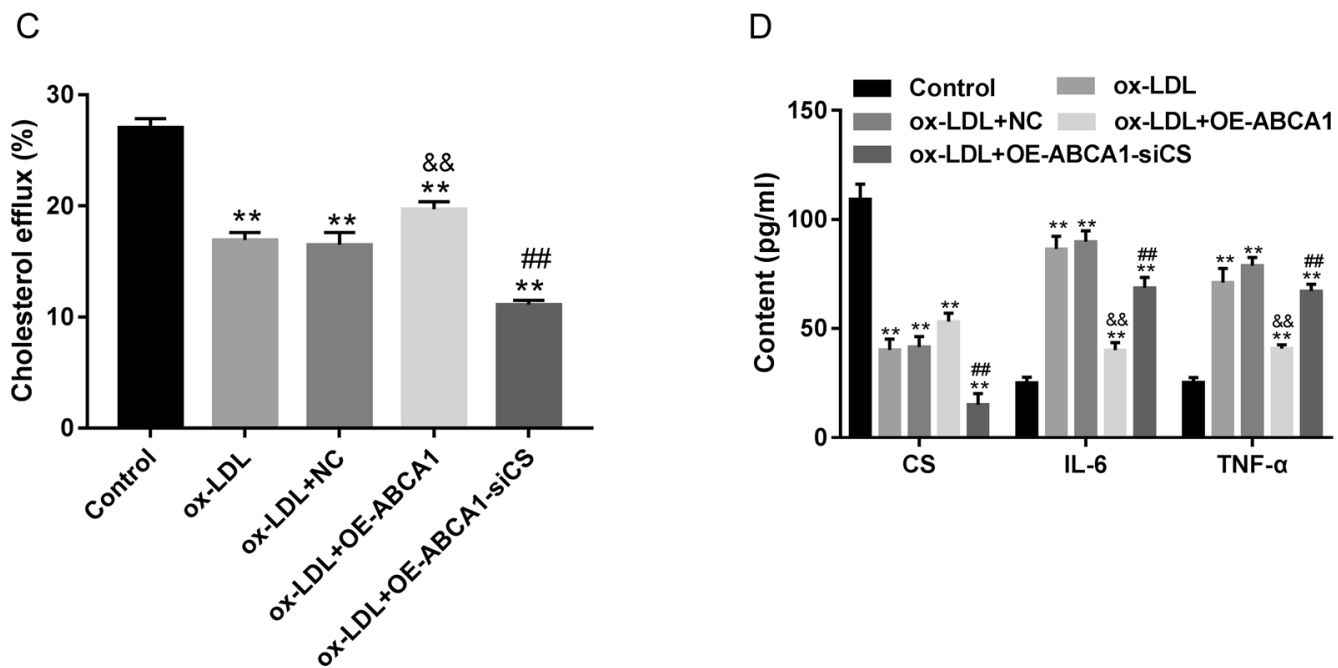

Fig. 8 Antagonistic effect of CS overexpression on ABCA1 inhibition. A, B the levels of ABCA1 and CS mRNA and protein expression in response to transfections. The original bands of WB are presented in Additional file 1: [2C]. C the cellular cholesterol efflux from VECs. D the levels of cellular CS, IL-6, and TNF-a in VECs. Ox-LDL, oxidized low-density lipoprotein. NC, overexpression negative control. ${ }^{* *} p<0.01$ vs. control by one-way ANOVA followed by Tukey's post hoc test. ${ }^{\&} \&<0.01$ vs. ox-LDL by one-way ANOVA followed by Tukey's post hoc test. ${ }^{\# \#} p<0.01$ vs. OE-ABCA1 by one-way ANOVA followed by Tukey's post hoc test. The above experiments were repeated three times and the mean value was obtained 


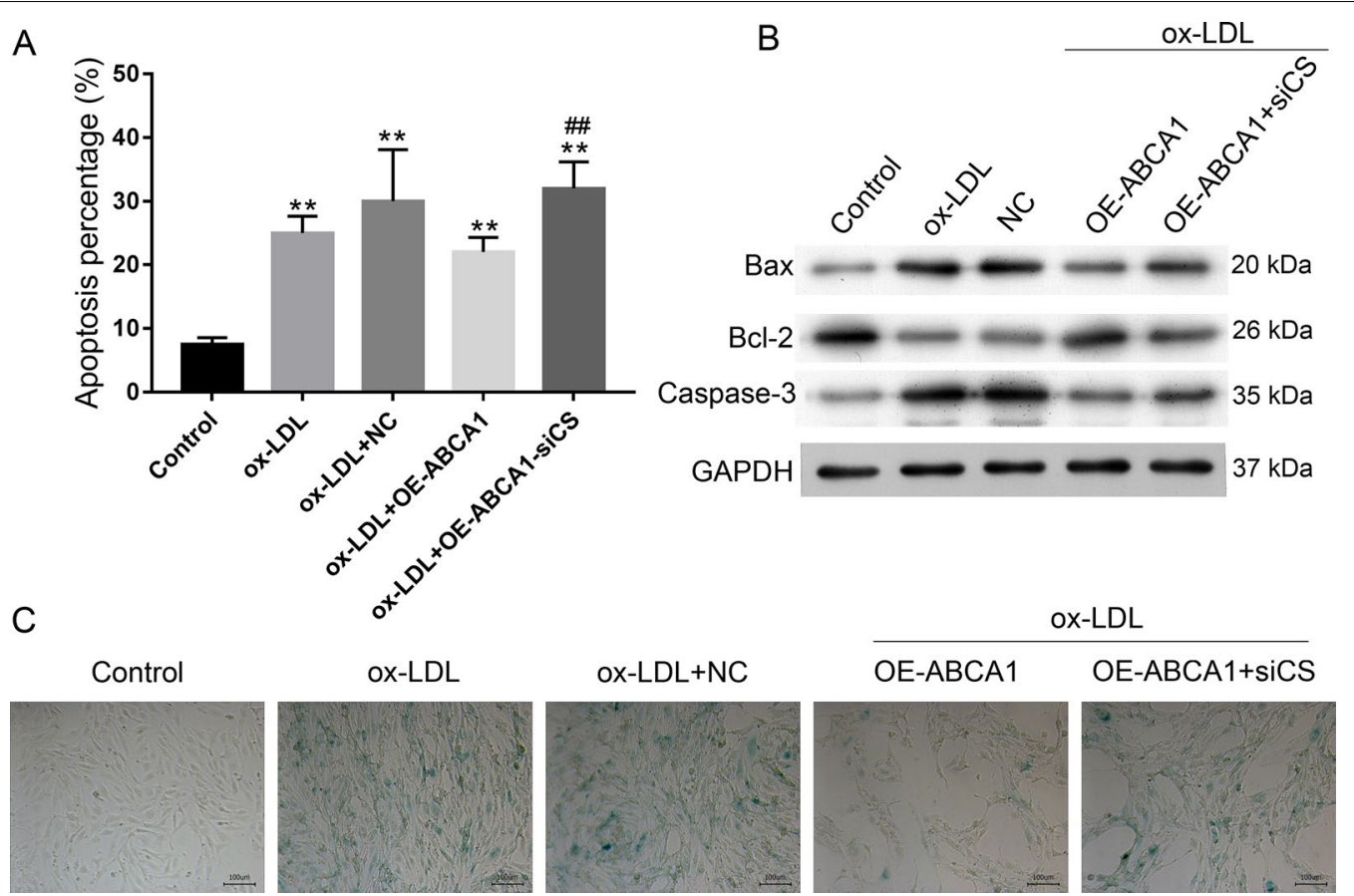

Fig. 9 CS inhibition promoted VEC apoptosis and aging. A The percentages of apoptotic VECs. B The expression of apoptosis-related proteins. The original bands of WB are presented in Additional file 1: [2D]. C The levels of senescence-associated $\beta$-galactosidase (SA- $\beta$-gal) activity in VECs. The repeated results were shown in Aditional file 2: Supplementary Figure 6. Ox-LDL, oxidized low-density lipoprotein. NC, overexpression negative control. ${ }^{* *} p<0.01$ vs. control by one-way ANOVA followed by Tukey's post hoc test. ${ }^{\# \#} p<0.01$ vs. OE-ABCA1 by one-way ANOVA followed by Tukey's post hoc test. The above experiments were repeated three times and the mean value was obtained

present study demonstrated the role played by the miR33-5p/ABCA1/CS axis in controlling cholesterol efflux in VECs. To the best of our knowledge, this is the first study to demonstrate the crucial role played by CS in cholesterol efflux in VECs.

ABCA1 is a direct target of miR-33-5p [14, 20]. Knockdown of miR-33-5p has been reported to decrease the levels of TC and TNF- $\alpha$, and promote cholesterol efflux from macrophage-derived foam cells [20]. Our present study demonstrated the targeted interaction that occurs between ABCA1 and miR-33-5p. ABCA1 is crucial for removing excess lipids by initiating the $\mathrm{RCT}$ process and promoting cholesterol efflux to ApoA1 [18]. Our present study demonstrated that overexpression of ABCA1 forced by treatment with an miR-33-5p inhibitor or transfection with an overexpression plasmid promoted cholesterol efflux in VECs. In addition, ABCA1 overexpression and the miR-33-5p inhibitor prevented ox-LDLinduced inflammation, apoptosis, and aging in VECs. These data suggested the potential therapeutic effect of ABCA1 expression and miR-33-5p downregulation in modulating lipid metabolism by promoting cholesterol efflux.
CS is essential for cell growth [26]. Our study also demonstrated the similar crucial roles played by CS and ABCA1 in regulating cholesterol efflux, inflammation, and apoptosis in VECs. We first confirmed that CS was a direct target of miR-33-5p, and then showed that overexpression of CS impeded the ox-LDL-induced aging and apoptosis of VECs. In addition, out data showed that the ox-LDL-induced reductions in cholesterol efflux in VECs could be rescued by CS overexpression. In contrast, inhibition of CS and ABCA1 both promoted VEC apoptosis. These results were inconsistent those reported by Alhindi et al. [22], who showed that lower levels of CS activity were associated with increased levels of cleaved caspase- 3 and lipotoxicity in $\mathrm{C} 1 \mathrm{C} 12$ muscle cells. A low level of CS expression is associated with cell apoptosis and oxidative stress [27]. CS catalyzes the citric acid cycle in mitochondria and can serve as a biomarker of mitochondrial function in mammals [26]. In aging cells, mitochondrial function declines [26, 28]. Our present study demonstrated that siRNAs for both CS and ABCA1 could promote VEC aging and apoptosis. CS functions downstream of $A B C A 1$ and was necessary for maintaining $\mathrm{ABCA1}$-mediated cellular functions and cholesterol 
efflux in VECs. These findings suggested CS as a key and novel mediator of ABCA1-mediated cholesterol efflux.

Citrate plays important roles in innate immunity and inflammation [29]. It was reported that the natural autoantibodies against CS (anti-CS IgM and IgG antibodies) were increased in rheumatoid arthritis (RA) patients treated with TNF inhibitors, certolizumab or etanercept therapy [30]. These antibodies might be associated with the clearance of apoptotic cells and restoration of immunological systems in the body [30]. Our study showed that inhibition of CS and ABCA1 increased the levels of IL- 6 and TNF- $\alpha$ in VECs, while the overexpression of CS and ABCA1 reduced the levels IL- 6 and TNF- $\alpha$. In addition, suppression of CS increased the levels of ABCA1reduced inflammatory cytokines (IL- 6 and TNF- $\alpha$ ) in VECs. These data suggested CS as a potent target for managing the inflammation-associated cholesterol efflux in VECs. However, the in vitro model used in our study has limitations, and an in vivo model such as apolipoprotein E-deficient (apoE(-/-)) hyperlipidemic mice or Abca1 binding site mutant (Abca1(BSM)) mice [31, 32] should to be used to further validate the role of the miR-33-5p/ ABCA1/CS axis in cholesterol efflux. Moreover, citrate synthase activity has been shown to decrease after myocardial infarction and be partially regulated by MMP-9 [33]. Furthermore, as MMP-9 has been shown to cleave citrate synthase ex vivo, the change and involvement of MMP-9 in lipid metabolism disorders should also be further investigated, along with the role played by the miR33-5p/ABCA1/CS axis.

\section{Conclusions}

In conclusion, our present study demonstrated the crucial roles played by the miR-33-5p/ABCA1/CS axis in regulating cholesterol efflux, inflammation, apoptosis, and aging in VECs. We also found that CS had similar functions to ABCA1 in controlling VEC behavior and cholesterol efflux. CS and ABCA1 expression in VECs promoted cholesterol efflux and inhibited ox-LDLinduced apoptosis, inflammation, and aging. Our rescue experiments confirmed that CS acts downstream of ABCA1. The miR-33-5p/ABCA1/CS axis plays pivotal roles in lipid metabolism and the underlying mechanisms of lipid metabolism disorders such as atherosclerosis and CVDs.

\footnotetext{
Abbreviations

CVDs: Cardiovascular diseases; ABCA1: ATP-binding cassette (ABC) transporter A1; CS: Citrate synthase; VECs: Vascular endothelial cells; ox-LDL: Oxidative lowdensity lipoprotein cholesterol; IL-6: Interleukin-6; TNF-a: Tumor necrosis factor a; TC: Total cholesterol; SA- $\beta$-gal: Senescence-associated $\beta$-galactosidase; HDL: High-density lipoprotein cholesterol; HMGCR: 3-Hydroxy-3-methylglutaryl coenzyme A reductasel; RCT: Reverse cholesterol transport; ApoA1: Apolipoprotein A I; ELISA: Enzyme-linked immunosorbent assay.
}

\section{Supplementary Information}

The online version contains supplementary material available at https://doi. org/10.1186/s12872-021-02228-7.

Additional file 1. The origin band of WB.

Additional file 2. Supplementary information of cells in multiple fileds.

\section{Acknowledgements}

We are very grateful to all the participants in our study.

\section{Authors' contributions}

FL conceived, designed, and planned the study. QX analyzed the data and statistics. JQP interpreted the data. QX prepared the manuscript. YG analyzed and searched the literature. FL collected the funds. All authors read and approved the final manuscript.

\section{Funding}

This study was supported by the Renshu Research and Development Fund of Hunan Provincial People's Hospital.

\section{Availability of data and materials}

The datasets used and analysed during the current study are available from the corresponding author on reasonable request.

\section{Declarations}

Ethical approval and consent to participate Not applicable.

\section{Consent for publication}

Not applicable.

\section{Competing interests}

All authors declare having no conflict of interest.

\section{Author details}

${ }^{1}$ Department of Cardiology, Hunan Provincial People's Hospital, The First Hospital Affiliated With Hunan Normal University, Changsha 410005, Hunan, People's Republic of China. ${ }^{2}$ Departments of Cardiovascular Surgery, The Second Xiangya Hospital of Central South University, middle Ren-Min Road No. 139, Changsha 410011, Hunan, People's Republic of China.

Received: 6 November 2020 Accepted: 24 August 2021

Published online: 13 September 2021

\section{References}

1. Tehrani DM, Zhao Y, Blaha MJ, Mora S, Mackey RH, Michos ED, Budoff MJ, Cromwell W, Otvos JD, Rosenblit PD. Discordance of low-density lipoprotein and high-density lipoprotein cholesterol particle versus cholesterol concentration for the prediction of cardiovascular disease in patients with metabolic syndrome and diabetes mellitus (from the Multi-Ethnic Study of Atherosclerosis [MESA]). Am J Cardiol. 2016;117(12):1921-7.

2. Abdullah SM, Defina LF, Leonard D, Barlow CE, Radford NB, Willis BL, Rohatgi A, McGuire DK, de Lemos JA, Grundy SM. Long-term association of low-density lipoprotein cholesterol with cardiovascular mortality in individuals at low 10-year risk of atherosclerotic cardiovascular disease: results from the Cooper Center Longitudinal Study. Circulation. 2018;138(21):2315-25.

3. Pedersen BK. Anti-inflammatory effects of exercise: role in diabetes and cardiovascular disease. Eur J Clin Invest. 2017;47(8):600-11.

4. Garcia-Arellano A, Ramallal R, Ruiz-Canela M, Salas-Salvadó J, Corella D, Shivappa N, Schröder H, Hébert J, Ros E, Gómez-Garcia E. Dietary inflammatory index and incidence of cardiovascular disease in the PREDIMED study. Nutrients. 2015;7(6):4124-38. 
5. Di Pietro N, Formoso G, Pandolfi A. Physiology and pathophysiology of oxLDL uptake by vascular wall cells in atherosclerosis. Vasc Pharmacol. 2016;84:1-7.

6. Tang $Y$, Jin X, Xiang Y, Chen Y, Shen C, Zhang Y, Li Y. The IncRNA MALAT1 protects the endothelium against ox-LDL-induced dysfunction via upregulating the expression of the miR-22-3p target genes CXCR2 and AKT. FEBS Lett. 2015;589(20PartB):3189-96.

7. Pérez L, Vallejos A, Echeverria C, Varela D, Cabello-Verrugio C, Simon F. OXHDL controls LOX-1 expression and plasma membrane localization through a mechanism dependent on NOX/ROS/NF-KB pathway on endothelial cells. Lab Invest. 2019;99(3):421.

8. Ou HC, Chou WC, Hung CH, Chu PM, Hsieh PL, Chan SH, Tsai KL. Galectin-3 aggravates Ox-LDL-induced endothelial dysfunction through LOX-1 mediated signaling pathway. Environ Toxicol. 2019;34:825-35.

9. Pothineni NVK, Karathanasis SK, Ding Z, Arulandu A, Varughese Kl, Mehta JL. LOX-1 in atherosclerosis and myocardial ischemia: biology, genetics, and modulation. J Am Coll Cardiol. 2017;69(22):2759-68.

10. Fazlali M, Kharazmi F, Kamran M, Malekzadeh K, Talebi A, Khosravi F, Soltani N. Effect of oral magnesium sulfate administration on lectin-like oxidized low-density lipoprotein receptor-1 gene expression to prevent atherosclerosis in diabetic rat vessels. J Diabetes Investig. 2019;10(3):650-8.

11. Lubrano V, Balzan S. Roles of LOX-1 in microvascular dysfunction. Microvasc Res. 2016;105:132-40

12. Xu Z, Dong A, Feng Z, Li J. Interleukin-32 promotes lipid accumulation through inhibition of cholesterol efflux. Exp Ther Med. 2017;14(2):947-52.

13. Zhao G-J, Tang S-L, Lv Y-C, Ouyang X-P, He P-P, Yao F, Chen W-J, Lu Q, Tang Y-Y, Zhang M. Antagonism of betulinic acid on LPS-mediated inhibition of $A B C A 1$ and cholesterol efflux through inhibiting nuclear factor-kappaB signaling pathway and miR-33 expression. PLoS ONE. 2013;8(9):e74782.

14. Chen W-M, Sheu WH, Tseng P-C, Lee T-S, Lee W-J, Chang P-J, Chiang A-N. Modulation of microRNA expression in subjects with metabolic syndrome and decrease of cholesterol efflux from macrophages via microRNA-33-mediated attenuation of ATP-binding cassette transporter A1 expression by statins. PLoS ONE. 2016;11(5):e0154672.

15. Karunakaran D, Dwevidi G, Rayner K. Macrophage cholesterol efflux and atherosclerosis in psoriasis: a role for microRNA-33. FASEB J. 2017;31(1):947.947.

16. Zhao G, Mo Z, Tang S, Ouyang X-P, He P, Lv Y, Yao F, Tan Y-I, Xie W, Shi J-F. Chlamydia pneumoniae negatively regulates ABCA1 expression via TLR2Nuclear factor-kappa B and miR-33 pathways in THP-1 macrophagederived foam cells. Atherosclerosis. 2014;235(2):519-25.

17. Zhang N, Lei J, Lei H, Ruan X, Liu Q, Chen Y, Huang W. MicroRNA-101 overexpression by IL-6 and TNF-a inhibits cholesterol efflux by suppressing ATP-binding cassette transporter A1 expression. Exp Cell Res. 2015;336(1):33-42.

18. Sasaki M, Komatsu T, Ikewaki K. Impact of hepatic ABCA1 (ATP-binding cassette transporter A1) deletion on reverse cholesterol transport a new clue in solving complex HDL (High-density lipoprotein) metabolism. Chicago: Am Heart Assoc; 2019.

19. Bashore AC, Liu M, Key CC, Boudyguina E, Wang X, Carroll CM, Sawyer JK, Mullick AE, Lee RG, Macauley SL, Parks JS. Targeted Deletion of Hepatocyte Abca1 Increases Plasma HDL (High-Density Lipoprotein) Reverse Cholesterol Transport via the LDL (Low-Density Lipoprotein) Receptor. Arterioscler Thromb Vasc Biol. 2019;39(9):1747-61.

20. Zhao L, Huang J, Zhu Y, Han S, Qing K, Wang J, Feng Y. miR-33-5p knockdown attenuates abdominal aortic aneurysm progression via promoting target adenosine triphosphate-binding cassette transporter
A1 expression and activating the PI3K/Akt signaling pathway. Perfusion. 2020;35(1):57-65.

21. Leandro JG, Espindola-Netto JM, Vianna MCF, Gomez LS, DeMaria TM, Marinho-Carvalho MM, Zancan P, Neto HAP, Sola-Penna M. Exogenous citrate impairs glucose tolerance and promotes visceral adipose tissue inflammation in mice. Br J Nutr. 2016;115(6):967-73.

22. Alhindi Y, Vaanholt LM, Al-Tarrah M, Gray SR, Speakman JR, Hambly C, Alanazi BS, Gabriel BM, Lionikas A, Ratkevicius A. Low Citrate Synthase Activity Is Associated with Glucose Intolerance and Lipotoxicity. J Nutr Metab. 2019;3(2019):8594825.

23. Wang K, Yang C, Shi J, Gao T. Ox-LDL-induced IncRNA MALAT1 promotes autophagy in human umbilical vein endothelial cells by sponging miR-216a-5p and regulating Beclin-1 expression. Eur J Pharmacol. 2019:858:172338.

24. Han XB, Li HX, Jiang YQ, Wang H, Li XS, Kou JY, Zheng YH, Liu ZN, Li H, Li J. Upconversion nanoparticle-mediated photodynamic therapy induces autophagy and cholesterol efflux of macrophage-derived foam cells via ROS generation. Cell Death Dis. 2017;8(6):2864.

25. Nie X, Liang L, Xi H, Jiang S, Jiang J, Tang C, Liu X, Liu S, Wan C, Zhao J. $2,3,7,8$-Tetrachlorodibenzo-p-dioxin induces premature senescence of astrocytes via WNT/ $\beta$-catenin signaling and ROS production. J Appl Toxicol. 2015;35(7):851-60.

26. Valentine JM, Li ME, Shoelson SE, Zhang N, Reddick RL, Musi N. NFkB Regulates Muscle Development and Mitochondrial Function. J Gerontol A Biol Sci Med Sci. 2020;75(4):647-53.

27. Cai Q, Zhao M, Liu X, Wang X, Nie Y, Li P, Liu T, Ge R, Han F. Reduced expression of citrate synthase leads to excessive superoxide formation and cell apoptosis. Biochem Biophys Res Commun. 2017;485(2):388-94.

28. Crupi AN, Nunnelee JS, Taylor DJ, Thomas A, Vit J-P, Riera CE, Gottlieb RA, Goodridge HS. Oxidative muscles have better mitochondrial homeostasis than glycolytic muscles throughout life and maintain mitochondrial function during aging. Aging (Albany NY). 2018;10(11):3327.

29. Williams NC, O'Neill LA. A role for the Krebs cycle intermediate citrate in metabolic reprogramming in innate immunity and inflammation. Front Immunol. 2018;9:141.

30. Simon D, Pusztai A, Najbauer J, Németh P, Szekanecz Z, Berki T. A8. 13 Natural autoantibodies against citrate synthase and DNA topoisomerase I in patients with rheumatoid arthritis and spondyloarthritis receiving anti-TNF-a therapy. London: BMJ Publishing Group Ltd; 2015.

31. Price NL, Rotllan N, Zhang X, Canfran-Duque A, Nottoli T, Suarez Y, Fernandez-Hernando C. Specific disruption of Abca1 targeting largely mimics the effects of miR-33 knockout on macrophage cholesterol efflux and atherosclerotic plaque development. Circ Res. 2019;124(6):874-80.

32. Miao $\mathrm{H}$, Zeng $\mathrm{H}$, Gong $\mathrm{H}$. microRNA-212 promotes lipid accumulation and attenuates cholesterol efflux in THP-1 human macrophages by targeting SIRT1. Gene. 2018;643:55-60.

33. de Castro Bras LE, Cates CA, DeLeon-Pennell KY, Ma Y, lyer RP, Halade GV, Yabluchanskiy A, Fields GB, Weintraub ST, Lindsey ML. Citrate synthase is a novel in vivo matrix metalloproteinase- 9 substrate that regulates mitochondrial function in the postmyocardial infarction left ventricle. Antioxid Redox Signal. 2014;21(14):1974-85.

\section{Publisher's Note}

Springer Nature remains neutral with regard to jurisdictional claims in published maps and institutional affiliations. 